\title{
FAST NAS-RIF ALGORITHM USING ITERATIVE CONJUGATE GRADIENT METHOD
}

\author{
A.M.Raid ${ }^{1}$, W.M.Khedr ${ }^{2}$, M. A. El-dosuky ${ }^{3}$ and Mona Aoud ${ }^{1}$ \\ ${ }^{1}$ Mansoura University, Faculty of Computer Science and Information System \\ ${ }^{2}$ Zagazig University, Faculty of Science \\ ${ }^{3}$ Mansoura University, Faculty of Computer Science and Information System
}

\begin{abstract}
Many improvements on image enhancemen have been achieved by The Non-negativity And Support constraints Recursive Inverse Filtering (NAS-RIF) algorithm. The Deterministic constraints such as non negativity, known finite support, and existence of blur invariant edges are given for the true image. NASRIF algorithms iterative and simultaneously estimate the pixels of the true image and the Point Spread Function (PSF) based on conjugate gradients method. NAS-RIF algorithm doesn't assume parametric models for either the image or the blur, so we update the parameters of conjugate gradient method and the objective function for improving the minimization of the cost function and the time for execution. We propose a different version of linear and nonlinear conjugate gradient methods to obtain the better results of image restoration with high PSNR.
\end{abstract}

\section{KEYWORDS}

blurred image, NAS-RIF algorithm, Image restoration, Point Spread Function (PSF), Conjugate Gradient (CG) and Peak Signal to Noise Ratio (PSNR).

\section{INTRODUCTION}

In image restoration field, A prior information such as the PSF of the imaging system and the observation noise [1]. When the blur effect is yielded on the objects, the PSF and the observation noise are unknown a priori. The identification and the restoration of the blurred images is a challenging problem in the world. The algorithm based on NAS-RIF [2,3] is proposed in order to identify and restore the images.

The scene which consists of a finite support object against a uniformly black, grey, or white background has been restored by The NAS-RIF technique. The restoration algorithm of NAS-RIF includes Recursive filtering of the blurred image to reduce a convex cost function.

The different versions of linear and nonlinear conjugate gradient methods are used for minimization of the NAS-RIF cost function. The conjugate gradient depends on gradient of an image at determine parameters. We update the CG's parameter and compare the results to 
Signal \& Image Processing : An International Journal (SIPIJ) Vol.5, No.2, April 2014

improve the restored image and the PSNR. The experimental results and comparison with the CG parameters show that the restoration effect is improved obviously.

The rest of this paper organize in three sections, The next section introduces fundamental of image restoration, section three explains NAS-RIF algorithm in more details with the update of $\mathrm{CG}$ parameters and section four shows the results of experimental.

\section{HISTORICAL REVIEW}

\subsection{Image Restoration}

Most of images can be modeled by using the standard degradation model as the following:

$$
g(x, y)=h(x, y) * f(x, y)+\eta(x, y)
$$

Where,

1- $(x, y)$ discrete pixel coordinate of the image

2- $g(x, y)$ image which has been blurred

3- $f(x, y)$ original image

4- $h(x, y)$ the point spread function (PSF)

5- $\eta(x, y)$ additive noise

6- $* 2 D$ convolution operator

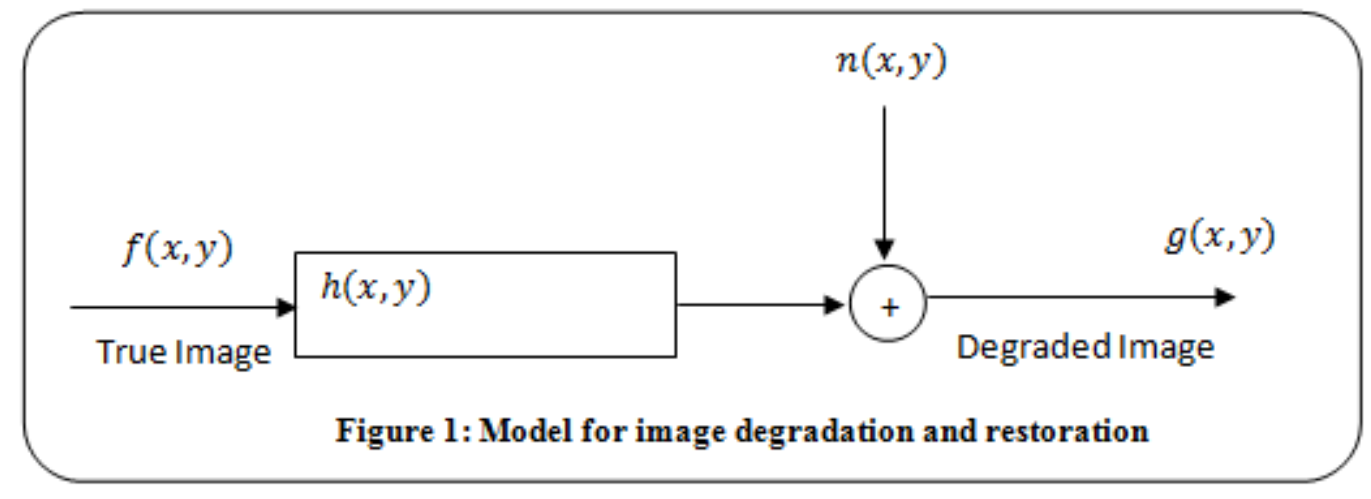

The above model shows that the degraded image $g(x, y)$, the original image $f(x, y)$, and the noise $\eta(x, y)$, are linearly combined and as a result the linear image restoration problem is the problem of retrieving the image from degraded image.

The form of bandwidth reduction of an ideal image owing to the imperfect image formation process is known as blurring. Relative motion between the camera and the original scene, or an optical system that is out of focus is caused this problem. Atmospheric turbulence, aberrations in the optical system and relative motion between the camera and the ground are caused blurs when aerial photographs are produced for remote sensing purposes. The optical images are not the only example of blurring, for example spherical aberrations of the electron lenses corrupted the electron micrographs, and X-ray scatter corrupted the CT scans suffer. 
Signal \& Image Processing : An International Journal (SIPIJ) Vol.5, No.2, April 2014

In addition to these blurring effects, any recorded image has been corrupted by noise. The medium may introduce the noise through which the image is created (random absorption or scatter effects), by the recording medium (sensor noise), by measurement errors because of the limited accuracy of the recording system, and by quantization of the data for digital storage.

The reconstruction or estimation of the uncorrupted image from a blurred and noisy one is concerned by the field of image restoration which also known as image deconvolution or image deblurring. Essentially, the inverse of the imperfections in the image formation system operation is performed on the image by the image restoration. A priori information are known about the characteristics of the degrading system and the noise in the use of image restoration methods. In practical situations, however, one may not be able to obtain this information directly from the image formation process. blur identification aims at estimating the attributes of the imperfect imaging system from the observed degraded image itself prior to the restoration process. The blind image deconvolution is combination of image restoration and blurs identification [4].

The purpose of the blind image deconvolution[5] is to obtain a reliable and effective estimate for the original image from the degraded version. To achieve this task, we use the partial information about the imaging process as a reference to deconvolve the true image and the PSF from the blurred image.

Existing blind deconvolution[6] methods include:

1) A priori blur identification methods.

2) Zero sheet separation methods.

3) ARMA parameter estimation methods.

4) Nonparametric methods.

A priori blur identification methods perform the blind deconvolution by identifying the PSF prior to the restoration. Typically, the PSF to be of a known parametric form is assumed by these methods. The unknown associated parameters must determine before restoration. For certain PSFs, the frequency-domain zeros of the PSFs determine the parameters uniquely. Once the zeros of $\mathrm{H}(\mathrm{u}, \mathrm{v})$ are identified, the parameters associated with $\mathrm{h}(\mathrm{x}, \mathrm{y})$ can be estimated accordingly. The a priori blur identification methods are relatively simple to achieve and require low computational complexity. But they have three major drawbacks. (1) They require the knowledge of the form of the PSF. (2) some PSFs (such as Gaussian PSF) simply don't have frequency zeros. (3) Additive noise can mask the frequency-domain nulls of and then degrade the performance. The lack of sufficient 2D polynomial algebra is one of the difficulty problems in the blind deconvolution.

The zero sheet separation algorithm [7] is such a factorization scheme. The valuable insight into the blind deconvolution problems in multiple dimensions is provided by the method of zero sheet separation. However, it suffers from several major problems. The First problemof this method is highly sensitive to noise. The Second problem, the method is prone to inaccuracy for larger images.

The true image as an autoregressive (AR) process and the PSF as a moving average (MA) process are modeled by Blind deconvolution using ARMA parameters estimation. The blurred image is therefore modeled as the autoregressive moving average (ARMA) process. The advantage of the ARMA parameter estimation methods is that they are less sensitive to noise because the models already take into account the noise. The major problem of these methods has the risk of ill-convergence to local minima. In addition, the total number of parameters cannot be 
Signal \& Image Processing : An International Journal (SIPIJ) Vol.5, No.2, April 2014

very large for practical computations. Usually, the restoration by these methods is not uniquely decided unless additional assumptions are made about the PSFs. They are therefore referred to as the nonparametric methods. Belongs to this category, there exist many algorithms including:

1) Iterative blind deconvolution (IBD) method.

2) Simulated Annealing (SA) method.

3) Nonnegativity and support constraints recursive inverse filtering (NAS-RIF).

In this paper, we consider the following details about the imaging process, the true image, and the PSF:

1- The linear model of the degradation as shown in Figure 1.

2- The grey, black, or white background of the image is uniformly.

3- A priori such as the nonnegative of the true image and its support; the smallest rectangle encompassing the object of interest known as the support (the region of support shown in Figure 2).

4- The inverse of the PSF exists, and both the PSF and its inverse are absolutely summable.

5- When the image background is black, the sum of all PSF pixels is assumed to be positive, which occurs in almost all image processing applications.
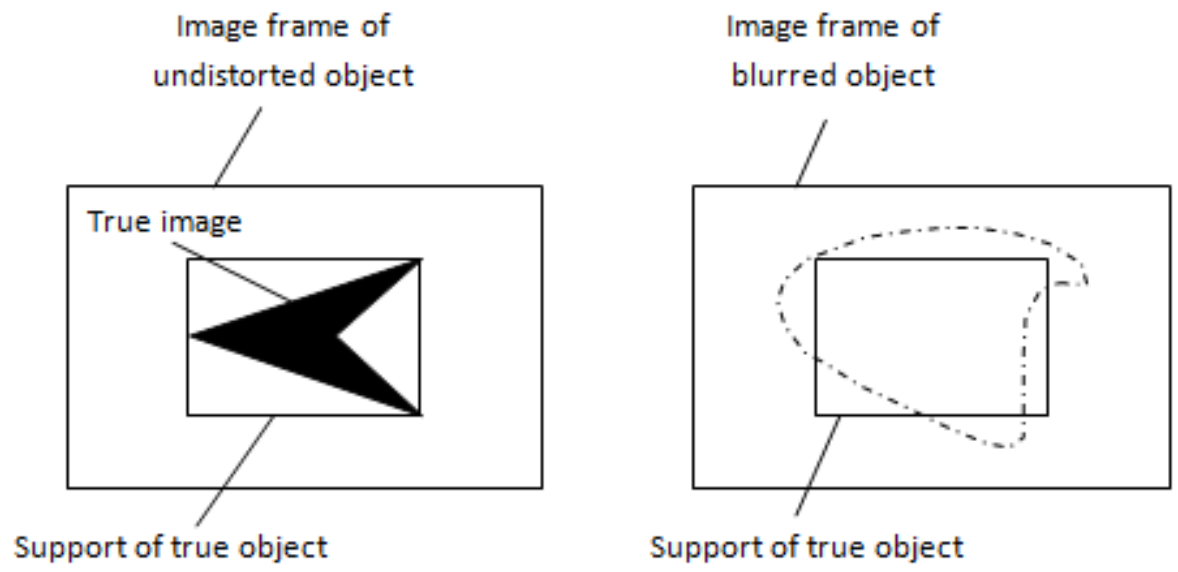

Figure 2: Model for image degradation and restoration process

\subsection{NAS-RIF Algorithm}

The assumption of non negativity on the true image is marked by NAS-RIF algorithm [8] that is, the algorithm supposes the true image pixel intensity values will be nonnegative. The knowledge of a known support is as well assumed. The only assumptions made on the PSF however, is that it is absolutely summable and that it has an inverse $h^{-1}(x, y)$ which is also absolutely summable. This method has an advantage is that it does not require the PSF to be of known finite extent, as do the other methods; this information is often difficult to get. The NAS-RIF algorithm is shown in Figure 3. It consists of a variable FIR Filter $\mathrm{u}(\mathrm{x}, \mathrm{y})$ with the blurred image $\mathrm{g}(\mathrm{x}, \mathrm{y})$ as input. The estimation of the true image $\hat{f}(x, y)$. Represents the output of this filter this estimate is passed through a nonlinear filter, which uses a non-expansive mapping to project the estimated image into the space representing the known characteristics of the true image. The error signal to update 
Signal \& Image Processing : An International Journal (SIPIJ) Vol.5, No.2, April 2014

the variable represents filter $\mathrm{u}(\mathrm{x}, \mathrm{y})$ the difference between the projected image $f_{N L}(x, y)$ and $f(x, y)$.

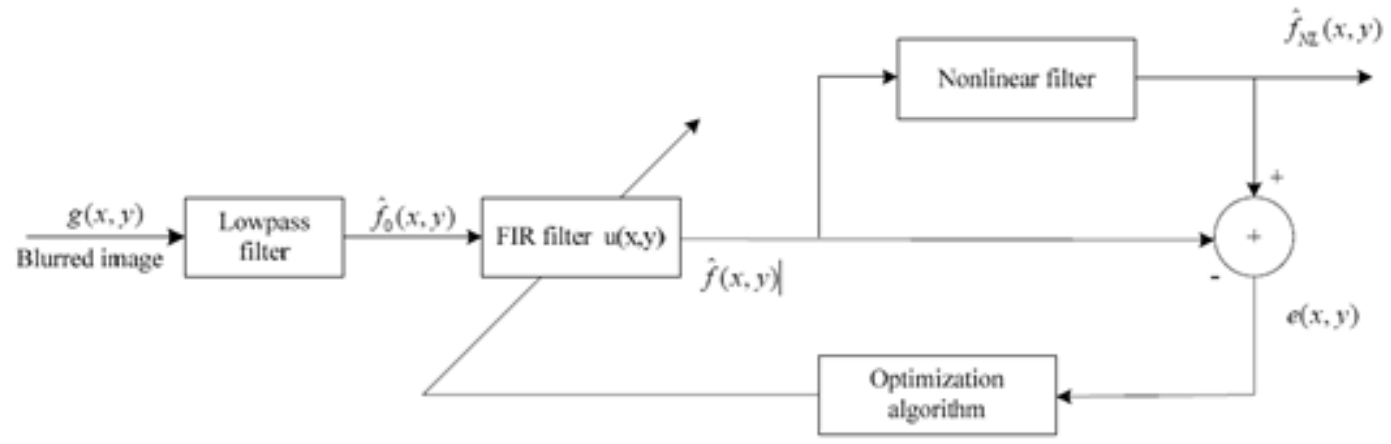

Figure 3: schema for blind image deconvolution method

If the image assumes to be nonnegative with known support, the NL block of Figure 3 shows the projection of the estimated image onto the set of images that are nonnegative with given finite support. Then, the negative pixel values within the region of support must be zero, and the background grey-level, $L_{B}$ is the pixel values outside the region of support. The cost function $J$ used in the restoration procedure is defined as:

$$
J=\sum_{\forall(x, y)}\left[\hat{f}_{N L}(x, y)-\hat{f}(x, y)\right]^{2}
$$

Where,

$$
\hat{f}_{N L}(x, y)=\left\{\begin{array}{cl}
L_{B} & \text { if }(x, y)<\bar{D}_{\text {up }} \\
0 & \text { if } f(x, y)<0 \text { and }(x, y) \in D_{\text {use }} \\
\hat{f}(x, y) & \text { if } f(x, y) \geq 0 \text { and }(x, v) \in D_{\text {un }}
\end{array}\right.
$$

Where $D_{\text {sup }}$ suppose to be the set of all pixels inside the region of support, and $\bar{D}_{\text {sup }}$ suppose to be the set of all pixels outside the region of support. Replace $\hat{\mathrm{f}}_{\mathrm{NL}}$ in equation (2) leads to

$$
J=\sum_{(x, y) \in D_{\text {sup }}}\left[\hat{f}(x, y)-L_{B}\right]^{2}+\sum_{(x, y) \in D_{\text {sup }}} \hat{f}^{2}(x, y)\left[\frac{1-\operatorname{sgn}(\hat{f}(x, y))}{2}\right]
$$

Where the $\operatorname{sgn}($.$) defined as$

$$
\operatorname{sgn}(f)= \begin{cases}-1 & \text { if } f<0 \\ 1 & \text { if } f \geq 0\end{cases}
$$

For globally minimizes $J$, The parameter $u(x, y)$ set to 0 for all $(x, y)$. This results to all black solution in which a restored image $\hat{f}(x ; y)=0$ for all $(x, y)$. To prevent this trivial solution, the sum of all the PSF coefficients assumed to be positive,i.e.

$$
\operatorname{sum}(h(x, y))>0
$$


Signal \& Image Processing : An International Journal (SIPIJ) Vol.5, No.2, April 2014

Also the filtering doesn't cause any loss of total image intensity because the sum of inverse filter coefficients is supposed to be 1 , the only method for constraining the parameters is normalized $u(x, y)$ at every iteration to fulfill this condition. Another method is add a third term to the cost function which known as a penalty method. The overall function is then represented by

$$
J=\sum_{(x, y) \in D_{\text {sup }}} \hat{f}^{2}(x, y)\left[\frac{1-\operatorname{sgn}(\hat{f}(x, y))}{2}\right]+\sum_{(x, y) \in D_{\text {sup }}}\left[\hat{f}(x, y)-L_{B}\right]^{2}+\gamma\left[\sum_{\forall(x, y)} u(x, y)-1\right]^{2}
$$

The cost function consists of three components. The negative pixels of the image estimate inside the region of support has been affected by The first component, the pixels of the image estimate outside the region of support that are not equal to the background color have been affected by the second component. The first component inhibits the pixels of the intermediate restorations from becoming highly negative. The detail algorithm is shows in Algorithm (1).

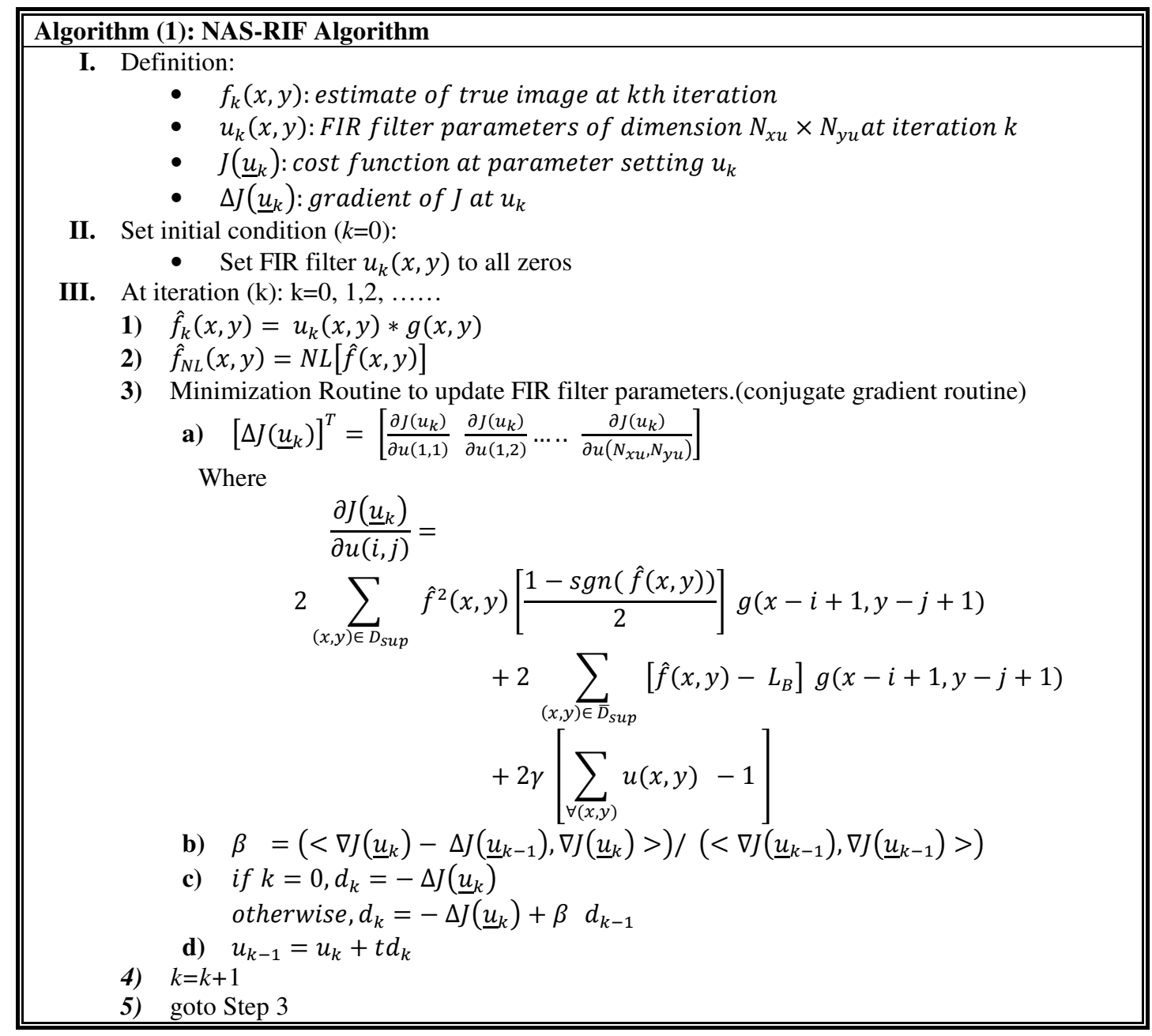


Signal \& Image Processing : An International Journal (SIPIJ) Vol.5, No.2, April 2014

We update the parameters of conjugate gradient [9] function and the value of constant $\gamma$ to be:

$$
\begin{gathered}
\beta_{k}=\left(<\nabla J\left(\underline{u}_{k}\right), \nabla J\left(\underline{u}_{k}\right)-\Delta J\left(\underline{u}_{k-1}\right)>\right) /\left(<\nabla J\left(\underline{u}_{k}\right), \nabla J\left(\underline{u}_{k-1}\right)>\right) \\
\gamma=\max \left(\beta_{k}\right)
\end{gathered}
$$

The algorithm is shows in Algorithm (2).

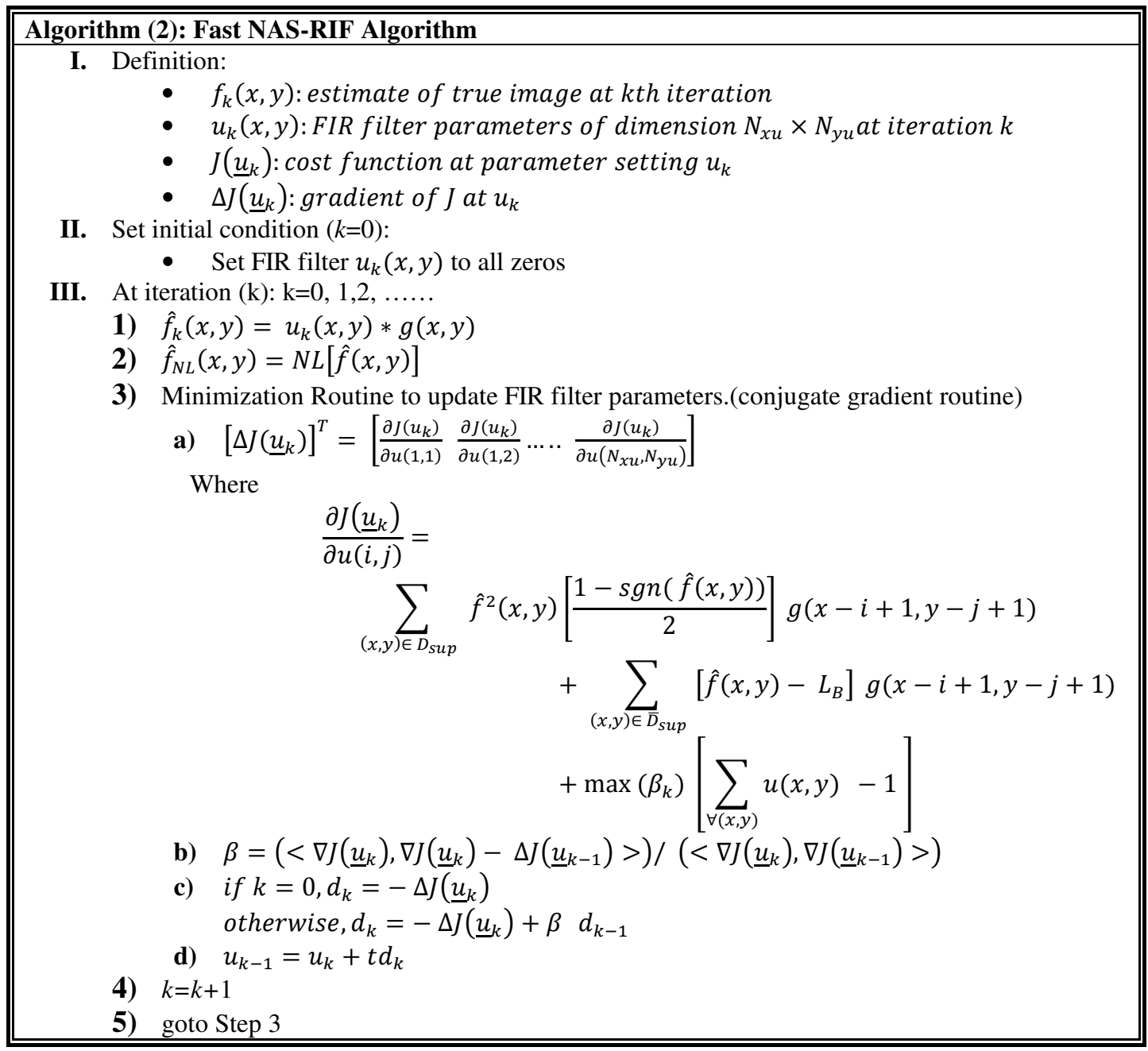

\section{SIMULATED RESULTS}

We implement the original and updated conjugate gradient parameter in the NAS-RIF algorithm, the objective function and compare the results on different Images samples with motion blur effect using length $=10$, angle $=0.05$, the experimental results show in the following figure in which the image restoration improve obviously. 
Signal \& Image Processing : An International Journal (SIPIJ) Vol.5, No.2, April 2014

\section{Example 1:}

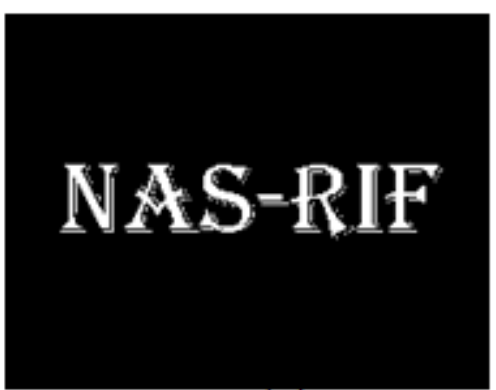

(a)

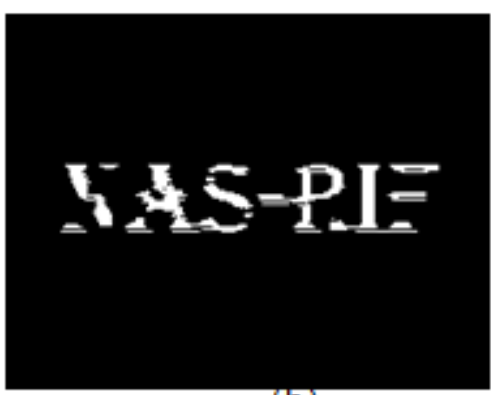

(b)

Figure 4.1: (a) Original Image

(b) Motion blurred image with length $=10$, angle $=0.05$

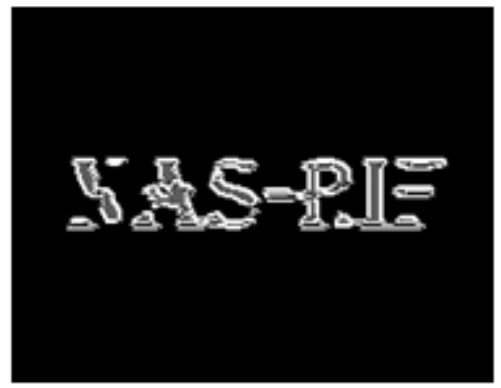

(a)

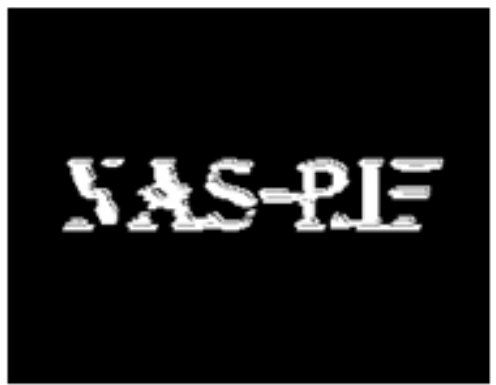

(b)

Figure 4.2: (a) Restored Image using NAS-RIF Algorithm, PSNR= $61.7979 \mathrm{~dB}$

(b) Restored Image using Fast NAS-RIF Algorithm, PSNR= $63.9548 \mathrm{~dB}$

\section{Example 2:}

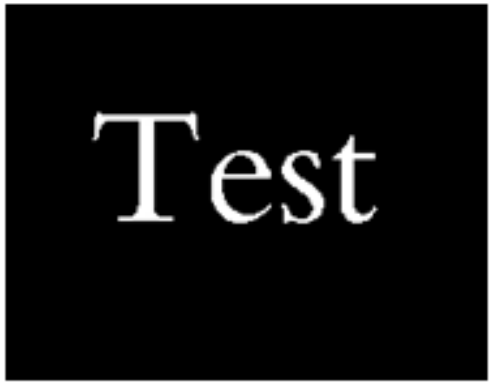

(a)

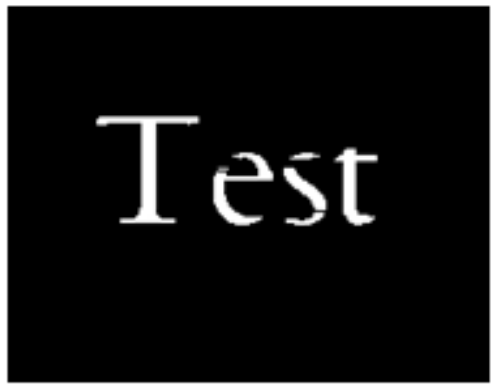

(b)

Figure 4.3: (a) Original Image (b) Motion blurred image with length $=10$, angle $=0.05$ 
Signal \& Image Processing : An International Journal (SIPIJ) Vol.5, No.2, April 2014

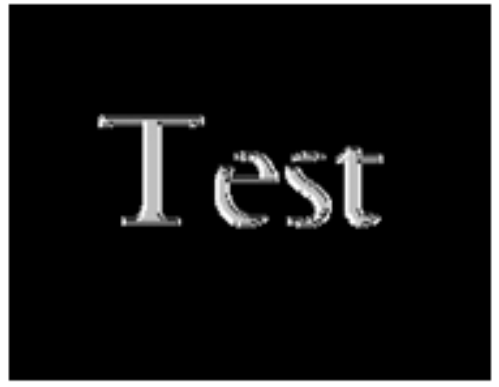

(a)

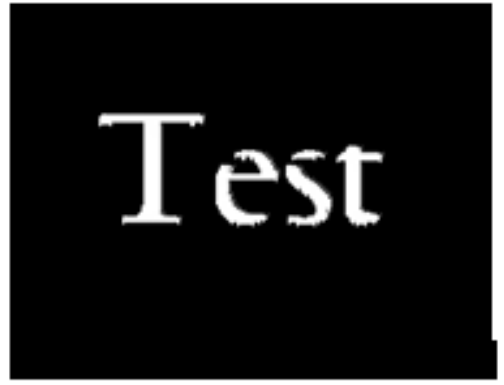

(b)

Figure 4.4: (a) Restored Image using NAS-RIF Algorithm, PSNR= $64.5471 \mathrm{~dB}$

(b) Restored Image using Fast NAS-RIF Algorithm, PSNR= $66.6734 \mathrm{~dB}$

\section{Example 3:}

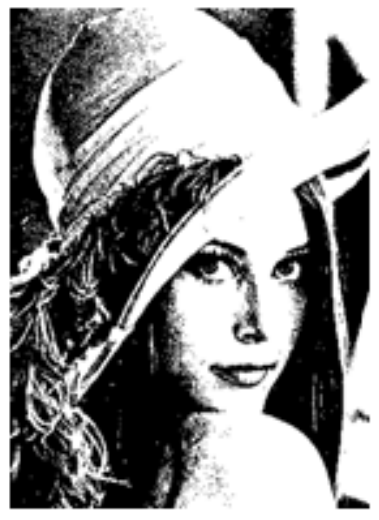

(a)

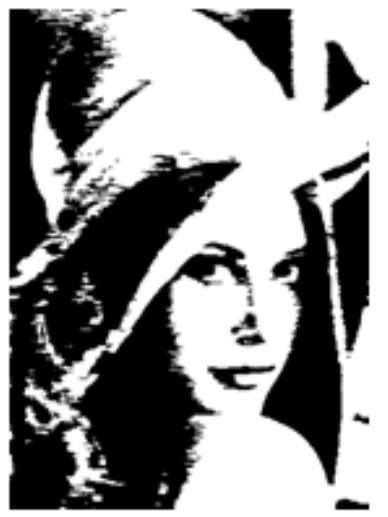

(b)

Figure 4.5: (a) Original Image

(b) Motion blurred image with length $=10$, angle $=0.05$

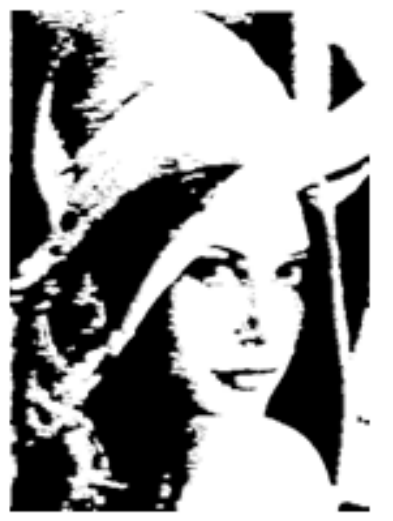

(a)

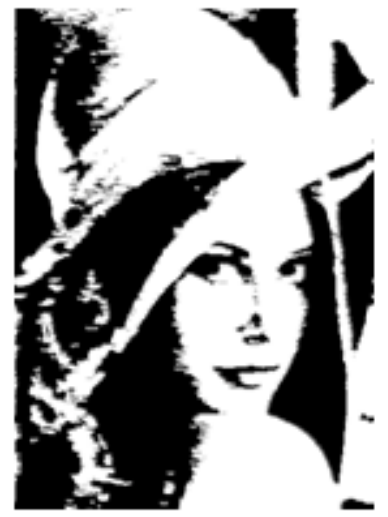

(b)

Figure 4.6: (a) Restored Image using NAS-RIF Algorithm, PSNR= 56.9118 dB

(b) Restored Image using Fast NAS-RIF Algorithm, PSNR $=57.3027 \mathrm{Db}$ 
Signal \& Image Processing : An International Journal (SIPIJ) Vol.5, No.2, April 2014

\section{CONCLUSION}

In this paper, the parameters of conjugate gradient method and the objective function are updated to improve the minimization of the cost function and the time for execution, and finally, a priori knowledge of the region of support of the object is changed to a simple classification procedure.

\section{REFERENCES}

[1] D. Kundur, D. Hatzinakos, "A Novel Blind Deconvolution Scheme for Image Restoration Using Recursive Filtering" [J], IEEE Transaction on Signal Processing, 46(2), 375-389(1998).

[2] Shirpur, D.Dhule, D.Maharastra, "Blind Image Restoration Methods", Department of Computer Engineering, R.C.Patel Institute of Technology, India, April 21, 2012

[3] B.Majhi, "Improvements in Blind Image Restoration", Department of Computer Science and Engineering National Institute of Technology Rourkela - 769 008, India, 2010

[4] D.Kundur and D.Hatzinakos, "Blind Image Deconvolution", IEEE Signal Processing Magazine, vol. 13 (3), pp. 43-64, May 1996.

[5] T.F.Chan, C.K.Wong, "Convergence of the alternating minimization algorithm for blind deconvolution", Linear Algebra and its Applications, Volume 316, Issues 1-3, 1 September 2000, Pages 259-285, ISSN 0024-3795.

[6] W.Wang, J.J.Zheng, S.Chen, S.Xu, H.Zhou, "Two-stage blind deconvolution scheme using useful priors", International Journal for Light and Electron Optics, Volume 125, Issue 4, February 2014, Pages 1503-1506, ISSN 0030-4026.

[7] P. Premaratne, C.C. Ko, "Retrieval of symmetrical image blur using zero sheets", Department of Electrical Engineering, The National University of Singapore, Singapore, Vision, Image and Signal Processing, Volume 148, Issue 1, February 2001.

[8] T.Xavier,'Development of image restoration techniques", National Institute of Technology Rourkela, May 2007.

[9] H. Yabe and M. Takano, "Global convergence properties of nonlinear conjugate gradient methods with modified secant condition", Computer Optim. Appl., 28, (2004), pp. 203-225. 